\title{
Buffer Behaviour in Massive MIMO Systems
}

\author{
Ranko Vojinović ${ }^{1}$, Svetlana Furtula ${ }^{2}$ \\ Assistant Professor, Faculty of Information Technology, Podgorica, Montenegro ${ }^{1}$ \\ Student, Faculty of Information Technology, Podgorica, Montenegro ${ }^{2}$
}

\begin{abstract}
In this paper, performances of buffers in Massive MIMO (Multiple-Input Multiple-Output) systems are analysed in the presence of statistical querying constraints. Querying constraints are included as limitations on the buffer capacity.
\end{abstract}

Keywords: Massive MIMO, buffer capacity, spatial multiplexing.

\section{INTRODUCTION}

In radio communications, multiple-input multiple-output (MIMO) is a method for multiplexing the capacity of a radio link using multiple transmit and receive antennas. MIMO has become an important element of wireless communication standards as IEEE 802.11n, HSPA+, LTE. One of essential parts of MIMO systems is spatial multiplexing [1]. In spatial multiplexing, a high-rate signals from different users are split into multiple lowerrate streams, and each stream is transmitted from different transmit antennas in the same frequency channel. Before multiplexing, input data packets may be temporarily stored in buffers. Buffers in this systems are used for many purposes, including interconnecting digital circuits operating at different rates, allowing synchronisations of packets which are multiplexed, and delaying the transfer time of packets in order to allow other operations to occur.

Massive MIMO is multiuser system where each base station is equipped with arrays of hundreds of antennas that are used in communication with one antenna terminals. This is one of the key technologies of the future $5 \mathrm{G}$ systems [2]. The main part of Massive MIMO systems is the relay technique. Relays give alternatives and independent paths for data transfer. By adding buffers to relays it is increased the spectral efficiency of the systems. Because of better understanding, firstly, we will consider a relay network with three nodes [3]-[4]. So, there is a source of information, a relay that is equipped with a buffer, and a destination. Considering that there is no direct path between the source and the destination, the communication is done through the relay. If the relay is selected to work as a receiver, then it receives data packets and stores them in the buffer. On the other side, if the relay is selected to work as a transmitter, it sends data packets to the destination. A spectral efficiency is increased two times by adding the buffer. There are multiple relay networks buffer-aided, but the essence is that incoming of data packets in the buffer is a random process, but they leave the buffer with a constant flow [5][6]-[7].

Buffer behaviour is analysed in this paper. Model of the system and its analysis is described in Section II. In Section III, results of the analysis are presented.

\section{SYSTEM MODEL}

Before spatial multiplexing to certain transmit antenna, data packets are stored in buffers with limited capacity $\mathrm{C}$. We assume that input flow packets which arrive from different sources are random, so, the statistics of incoming packets in system are treated as Poisson process. This model perfectly describes transfer of larger data flows which is often case in practice.

We assume that a service discipline of a transmitter is such that buffers empty with constant flow. This assumption may well approximate the spatial multiplexing of packets that are buffered and forward them to transmit antennas.

Assume that in the buffer can be stored $\mathrm{C}$ packets, where "zero" packet is included, respectively, a case when the buffer is empty. If the buffer is full, all packets that arrive will be lost, respectively:

$\mathrm{P}(\mathrm{k}=\mathrm{C})=0$

where $P(k=C)=S c$ is probability that the buffer has $C$ packets, so, $P(k=n)=S n$ is probability that the buffer has $\mathrm{n}$ packets. The arrival of packets in the buffer is a stationary stochastic process, so we have:

$\mathrm{S}_{\mathrm{n}}^{-}=\mathrm{S}_{\mathrm{n}-1}^{+}$

where $\mathrm{S}_{\mathrm{n}}^{-}$is probability that in the buffer there are $\mathrm{n}$ packets just before leaving a multiplexed packet. $\mathrm{S}_{\mathrm{n}-1}^{+}$is a probability that in the buffer there are n-1 packets just after leaving the packet.

According to the initial assumption, packets that arrive in buffer have Poisson distribution. If $\mathrm{P}_{\mathrm{A}}(\mathrm{k})$ is a probability of arrival of $\mathrm{k}$ packets in a certain time interval, then, we have:

$P_{A}(k)=\frac{\lambda^{k} e^{-\lambda}}{k !}$

where $\lambda$ is expected number of packets in the interval.

Equations that describe buffer state in a moment when a packet is in a multiplexing queue are:

$\mathrm{S}_{1}=\mathrm{S}_{0} \mathrm{P}_{\mathrm{A}}(1)+\mathrm{S}_{1} \mathrm{P}_{\mathrm{A}}(0)$

$S_{2}=S_{0} P_{A}(2)+S_{1} P_{A}(1)+S_{2} P_{A}(0)$ 


$$
\begin{gathered}
1-\lambda(1-\mathrm{L})=\mathrm{S}_{0} \mathrm{P}_{\mathrm{A}}(\mathrm{k}=0) \\
\mathrm{P}_{\mathrm{A}}(\mathrm{k}=0)=\mathrm{e}^{-\lambda}
\end{gathered}
$$

After calculating equations (12) and (11), we have:

$$
\mathrm{L}=1+\frac{\mathrm{S}_{0} \mathrm{e}^{-\lambda}-1}{\lambda}
$$

The relation (13) is an analytically depending expression of the expected number of packets that are lost because of overdraft capacity of the buffer.

$\mathrm{S}_{\mathrm{n}-1}=\sum_{\mathrm{k}=0}^{\mathrm{n}} \mathrm{S}_{\mathrm{k}} \mathrm{P}_{\mathrm{A}}(\mathrm{n}-\mathrm{k})$, for $2 \leq \mathrm{n} \leq \mathrm{C}-1$

The relation (5) is a recursive form of equation buffer state.

For n-1, we have:

$S_{0}=S_{0} P_{A}(1)+S_{1} P_{A}(0)+S_{0} P_{A}(0)$

where $S_{0} P_{A}(0)$ is the probability that the buffer is empty.

Probabilities of states of systems $\mathrm{S}_{0}, \mathrm{~S}_{1}, \ldots \mathrm{S}_{\mathrm{c}-1}$ are mutually independent and they make a complete group of events, so, we have:

$\sum_{\mathrm{k}=0}^{\mathrm{C}-1} \mathrm{~S}_{\mathrm{k}}=1$

Using the recursive formula (5) and equations (6) and (7), we get equations of buffer state in mathematical form:

$$
\begin{gathered}
\left(\begin{array}{cccc}
1 & 1 & 1 & 1 \\
\mathrm{P}_{\mathrm{A}}(0)+\mathrm{P}_{\mathrm{A}}(1)-1 & \mathrm{P}_{\mathrm{A}}(0) & 0 & 0 \\
\mathrm{P}_{\mathrm{A}}(2) & \mathrm{P}_{\mathrm{A}}(1)-1 & \mathrm{P}_{\mathrm{A}}(0) & 0 \\
\cdot & \cdot & \cdot & \cdot \\
\cdot & \cdot & \cdot & \cdot \\
\cdot & \cdot & \cdot & \cdot \\
\mathrm{P}_{\mathrm{A}}(\mathrm{C}-1) & \mathrm{P}_{\mathrm{A}}(\mathrm{C}-2) & \mathrm{P}_{\mathrm{A}}(\mathrm{C}-3) & \mathrm{P}_{\mathrm{A}}(0)
\end{array}\right) \\
\left(\begin{array}{c}
\mathrm{S}_{0} \\
\mathrm{~S}_{1} \\
\mathrm{~S}_{2} \\
\cdot \\
\cdot \\
\mathrm{S}_{\mathrm{C}-1}
\end{array}\right)=\left(\begin{array}{c}
1 \\
0 \\
0 \\
\cdot \\
\cdot \\
\cdot \\
0
\end{array}\right)
\end{gathered}
$$

Also, we will determine the capacity of the buffer, depending on traffic intensity or depending on the preset allowed loses.

$\mathrm{L}$ is expected number of packets that have been lost because of exceeding the buffer size.

Effective throughput will be:

$\mathrm{T}=\lambda(1-\mathrm{L})$

In this case, $\mathrm{T}$ is the probability that the received packed will be multiplexed.

The probability of opposite event is:

$\overline{\mathrm{T}}=1-\lambda(1-\mathrm{L})$

In the observed interval, the packet will not be multiplexed when the buffer is empty or when in the same interval does not have incoming packets, so, we have:

\section{III.RESULTS ANALYSIS}

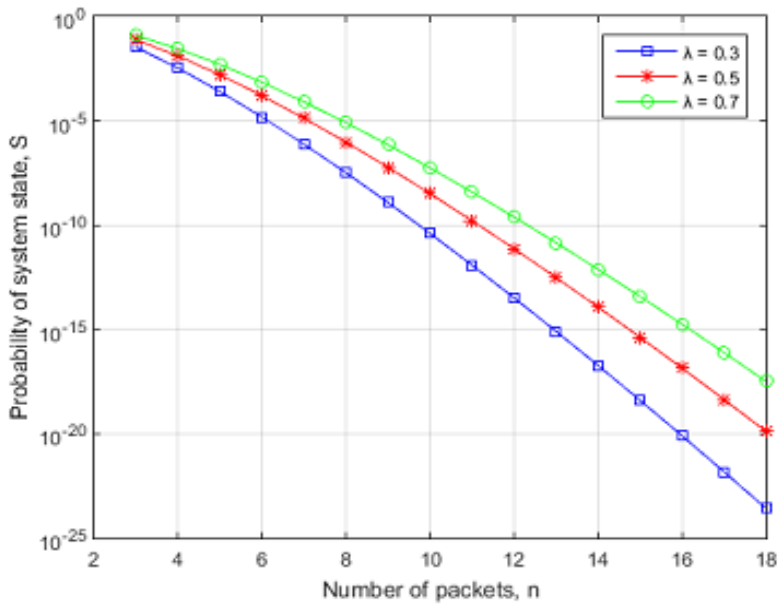

Fig. 1 The probability of the system state depending on number of packets

Fig.1. shows the probability of the system state $S$ depending on numbers of packets $\mathrm{n}$ for the chosen values of $\lambda=0.3, \lambda=0.5$ and $\lambda=0.7$. It can be observed, the probability of the system state decreases monotonously with increasing of the number of packets.

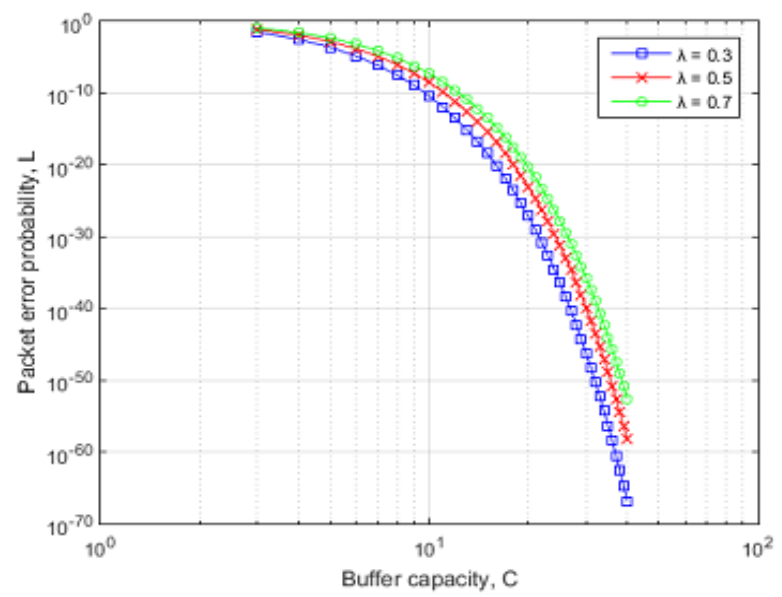

Fig 2. The packet error probability depending on the capacity of the buffer due to the buffer capacity distortion capacity of the buffer due to the buffer capacity distortion for the chosen values of the $\lambda=0.3, \lambda=0.5$ and $\lambda=0.7$. It can be seen, the packet error probability decrees with increasing of the buffer capacity.
Fig. 2. shows the packet error probability depending on the 


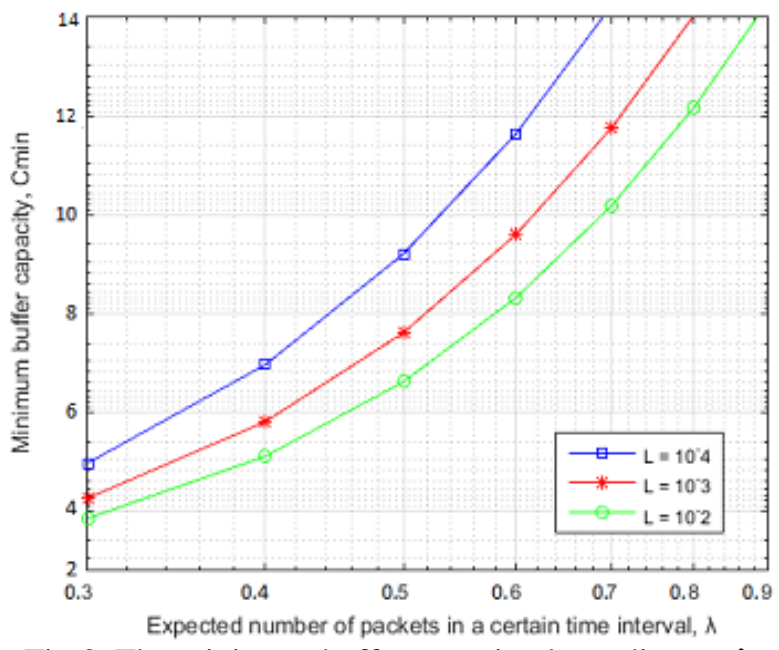

Fig.3. The minimum buffer capacity depending on $\lambda$
From 1996 to 2006, he was with the Maritime faculty at the University of Montenegro. In 2007 he joined the Faculty of Information Technologies of the University "Mediterranean" (Montenegro), where he is currently an Assistant Professor, teaching several courses in computer networks. His main research interests are in telecommunications and modelling and analysis of network protocols.

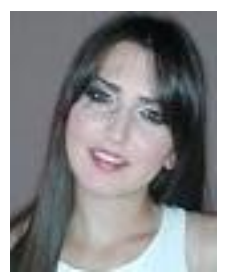

Svetlana Furtula received B.Sc. and Spec.Sc. degrees in Information Technologies from University of Mediterranean, Montenegro, in 2013 and 2014, respectively. She was an exchange master student in Telecommunications at University of L'Aquila, Italy, in 2015. Currently, she does her master thesis and she works in a Department of

The minimum buffer capacity depending on $\lambda$ for chosen Telecommunications in Montenegro Police Directorate. values $\mathrm{L}=10^{-2}, \mathrm{~L}=10^{-3}, \mathrm{~L}=10^{-4}$, is presented in Fig.3. Her main research interests are in telecommunication The minimum capacity of the buffer increases with networks. increasing of the number of expected packets.

\section{IV.CONCLUSION}

This paper presents the results obtained by analyzing performances of buffers in the presence of statistical querying constraints that are included as limitations on the buffer capacity. Using a simulation model, realized in MATLAB, and the theoretical analysis confirm the validity of the theoretical considerations.

\section{REFERENCES}

[1] Andreas F. Molisch and Moe Z. WinSadk, MIMO Systems with Antenna Selection", IEEE Microwave magazine, March 2004.

2] Erik G Larsson, Ove Edfors, Fredrik Tufvesson and Thomas L. Marzetta, "Massive MIMO for Next Generation Wireless Systems", IEEE Communications Magazine, (52), 2, 186-195, 2014.

[3] B. Xia, Y. Fan, J. Thompson, and H. V. Poor, "Buffering in a three node relay network," IEEE Trans. Wireless Commun., vol. 7, no. 11, pp. 4492 - 4496, Nov. 2008. 2.

[4] N. Zlatanov and R. Schober, "Buffer-Aided Relaying with Adaptive Link Selection — Fixed and Mixed Rate Transmission," IEEE Trans. Info. Theory, vol. 59, no. 5, pp. 2816-40.3, May 2013.

[5] Su Min Kim and Mats Bengtsson, "Virtual Full-Duplex BufferAided Relaying in the Presence of Inter-Relay Interference ", Jan 2016.

[6] N. Nomikos, D. Vouyioukas, T. Charalambous, I. Krikidis, D. N. Skoutas, and M. Johansson, "Capacity Improvement through Buffer-Aided Successive Opportunistic Relaying “, June 2013.

[7] Su Min Kim and Mats Bengtsson "Virtual Full-Duplex BufferAided Relaying - Relay Selection and Beamforming “, June 2013.

\section{BIOGRAPHIES}

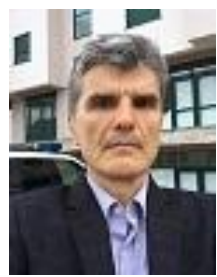

Ranko Vojinović received the B.Sc. degree in Electrical Engineering from University of Montenegro in 1982, and the M.Sc. and Ph.D. degrees from University of Belgrade in 1992 and 2003, respectively. Since 1982 he has worked in a Department of Telecommunications in Montenegro Police Directorate. 